\title{
A matrix approach for generalizing two curious divisibility properties
}

\author{
Emrah Kiliç
}




\title{
A MATRIX APPROACH FOR GENERALIZING TWO CURIOUS DIVISIBILITY PROPERTIES
}

\author{
EMRAH KILIÇ
}

Received 21 February, 2012

\begin{abstract}
We shall consider two curious divisibility properties due to [1,5]. Our main purpose is to generalize these properties for a general second order linear recursion. We use generating matrix approach for our purposes. By using our results, we derive a new recursive identities for two general second order linear recurrences. We give a more general result extending earlier divisibility properties on the similar subject.
\end{abstract}

2000 Mathematics Subject Classification: 11B37; 15B36

Keywords: binary linear recursions, divisibility property, matrix methods

\section{INTRODUCTION}

The Fibonacci numbers have many interesting properties in accordance with their nature and the recursively definition. We shall be interested in their divisibility properties along the paper. We recall that

$$
\operatorname{gcd}\left(F_{n}, F_{m}\right)=F_{\operatorname{gcd}(n, m)}
$$

and

$$
F_{k n} \text { is a multiple of } F_{n} \text {. }
$$

An extension of these divisibility properties was given showing Matijasevich [4] as that if $n>2$, the Fibonacci number $F_{m}$ is a multiple of $F_{n}^{2}$ if and only if $m$ is multiple of $n F_{n}$. This fact and its detailed proof was also given in [2] (pp. 294) showning

$$
F_{k n} \equiv k F_{n} F_{n+1}^{k-1} \text { and } F_{k n+1} \equiv F_{n+1}^{k}\left(\bmod F_{n}^{2}\right) \text {. }
$$

The above fact and the following similar results can also be found in [1,5] :

i) $F_{k n-1}-F_{n-1}^{k}$ is divisible by $F_{n}^{2}(n, k=1,2, \ldots)$,

ii) $F_{k n-2}-(-1)^{k+1} F_{n-2}^{k}$ is divisible by $F_{n}^{2}(n, k=1,2, \ldots)$.

We mainly consider these two curious properties and then we generalize them to a general second order linear recursion $\left\{U_{n}(p)\right\}$, or briefly $U_{n}$, defined for $n>1$ by

$$
U_{n}(p)=p U_{n-1}(p)+U_{n-2}(p),
$$

with initials $U_{0}(p)=0$ and $U_{1}(p)=1$. 
The generalized Lucas sequence $\left\{V_{n}(p)\right\}$, or briefly $V_{n}$, is defined by

$$
V_{n}(p)=p V_{n-1}(p)+V_{n-2}(p),
$$

with initials $V_{0}(p)=2, V_{1}(p)=p$.

The Binet forms of them are

$$
U_{n}=\frac{\alpha^{n}-\beta^{n}}{\alpha-\beta} \text { and } V_{n}=\alpha^{n}+\beta^{n},
$$

where $\alpha, \beta=\left(p \pm \sqrt{p^{2}+4}\right) / 2$.

Throughout the paper we consider the properties

a) $U_{k n-1}-U_{n-1}^{k}$ is divisible by $U_{n}^{2}(n, k=1,2, \ldots)$,

b) $p^{k-1} U_{k n-2}-(-1)^{k+1} U_{n-2}^{k}$ is divisible by $U_{n}^{2}(n, k=1,2, \ldots)$.

Matijasevich and Cavachi proved the properties (i) and (ii) in [2,5] but the quotients

$$
\frac{F_{k n-1}-F_{n-1}^{k}}{F_{n}^{2}} \text { and } \frac{F_{k n-2}-(-1)^{k+1} F_{n-2}^{k}}{F_{n}^{2}}
$$

were not considered. It seems that to find the quotients are not easy.

However, we shall focus on quotients arising from (a) and (b) :

$$
\frac{U_{k n-1}-U_{n-1}^{k}}{U_{n}^{2}} \text { and } \frac{p^{k-1} U_{k n-2}-(-1)^{k+1} U_{n-2}^{k}}{U_{n}^{2}}
$$

for all $n$ and $k$ such that $n, k \geq 1$.

Matrix methods are essential tools for solving problems arising from number theory, for more details see [3].

Our approach is to use matrix methods for deriving these quotients explicitly. We first look at values of the quotients for fixed $n$ and increasing values of $k$ starting from 1 and then find a generating matrix for these values for fixed $n$ and increasing values of $k$. After we find the generating matrices for these quotients, we will give two explicit statements for them. Using these two explicit statements, we obtain two new recursive identities for the general second order linear recurrences. Finally by considering and extending these two recursive identities, we find new recursive identities for the general second order linear recursions. In order to guarantee the accuracy of matrix computations, all the equalities appearing in this paper have been verified through "Maple" commands.

\section{General Cases}

We start with the first quotient with the case $p=1, U_{n}=F_{n}$ ( $n$th Fibonacci numbers) and its values for some $n$ and $k$ are shown in Table 1.

We will see that the entries of each row in Table 1 have a generating matrix. For example, the entries of the third row of Table $1,\{1,5,22,94,399,1691, \ldots\}$, could be generated by powers of a matrix. Continuing this process each row entries in each 
TABLE 1 .

\begin{tabular}{l|l|l|l|l|l|l|l|l}
\multicolumn{10}{c}{$\left(F_{k n-1}-F_{n-1}^{k}\right) / F_{n}^{2}$} \\
$n \backslash k$ & 1 & 2 & 3 & 4 & 5 & 6 & 7 & $\ldots$ \\
\hline 1 & 0 & 1 & 1 & 2 & 3 & 5 & 8 & $\ldots$ \\
\hline 2 & 0 & 1 & 4 & 12 & 33 & 88 & 232 & $\ldots$ \\
\hline 3 & 0 & 1 & 5 & 22 & 94 & 399 & 1691 & $\ldots$
\end{tabular}

table can be obtained from the quotients for all $n, k$ and $p$. We will state this fact in general by matrix methods.

Before this process, we need some auxiliary results and definitions.

Define a second order linear sequence $\left\{A_{n}(p)\right\}$, or briefly $A_{n}$, for $n>1$ as

$$
A_{n}=p A_{n-1}+A_{n-2}
$$

with initials $A_{0}=p$ and $A_{1}=p^{2}+3$.

From the definitions of sequences $\left\{U_{n}\right\}$ and $\left\{A_{n}\right\}$, we have that

$$
A_{n}=U_{n+2}+U_{n}=V_{n}+U_{n-1} .
$$

Denote the first quotient by $s(n, k, p)$, or briefly $s(k, p)$ :

$$
s(n, k)=\left(U_{k n-1}-U_{n-1}^{k}\right) / U_{n}^{2} .
$$

Define two matrices $H(n, p)$, or briefly $H(n)$, and $G(n, k, p)$, or briefly $G(n, k)$, of order 3 as shown:

$$
H(n)=\left[\begin{array}{ccc}
A_{n-1} & -U_{n-1} V_{n}+(-1)^{n+1} & (-1)^{n} U_{n-1} \\
1 & 0 & 0 \\
0 & 1 & 0
\end{array}\right]
$$

and

$$
G(n, k)=\left[\begin{array}{ccc}
s(n, k+2) & t(n, k+2) & (-1)^{n} U_{n-1} s(n, k+1) \\
s(n, k+1) & t(n, k+1) & (-1)^{n} U_{n-1} s(n, k) \\
s(n, k) & t(n, k) & (-1)^{n} U_{n-1} s(n, k-1)
\end{array}\right],
$$

where

$$
t(n, k)=\frac{U_{k n} U_{n-1}^{2}-U_{(k-2) n}-U_{2 n} U_{n-1}^{k}}{U_{n}^{3}} .
$$

Lemma 1. For $n \geq 1$, the eigenvalues of the matrix $H(n)$ are $\alpha^{n}, \beta^{n}$ and $U_{n-1}$.

Proof. The characteristic polynomial of $H(n)$ is

$$
x^{3}-A_{n-1} x^{2}+x\left(U_{n-1} V_{n}+(-1)^{n}\right)-(-1)^{n} U_{n-1}
$$

and it is factorized as $\left(x-\alpha^{n}\right)\left(x-\beta^{n}\right)\left(x-U_{n-1}\right)$, as claimed.

Now we state one of our main two results: 
Theorem 1. For $n \geq 1$,

$$
H(n)^{k}=G(n, k) .
$$

Proof. To prove the claim, it is sufficient to verify the followings:

$$
s(n, k+1) A_{n-1}+t(n, k)=s(n, k+2)
$$

and

$$
s(n, k+1)\left(-U_{n-1} V_{n}+(-1)^{n+1}\right)+(-1)^{n} U_{n-1} s(n, k)=t(n, k+2) .
$$

For proving them, it is enough to consider the definitions of $s(n, k)$ and $t(n, k)$. The above equations give us the following matrix equation:

$$
G(n, k-1) H(n)=G(n, k) .
$$

Since $G(n, 1)=H(n)$, we deduce that $G(n, k)=H(n)^{k}$, as claimed.

We see that the powers of matrix $H(n)$ generate the first quotient and since the matrix has integer entries, it is clear that the first quotient are integers.

Since all eigenvalues of $H(n)$ are different from each other, we diagonalize it. $H(n)$ is a companion matrix so that it is diagonalized with the Vandermonde matrix

$$
V=\left[\begin{array}{ccc}
\alpha^{2 n} & \beta^{2 n} & U_{n-1}^{2} \\
\alpha^{n} & \beta^{n} & U_{n-1} \\
1 & 1 & 1
\end{array}\right],
$$

as $V^{-1} H(n) V=D$, where $D$ is the diagonal matrix $D=\operatorname{diag}\left(\alpha^{n}, \beta^{n}, U_{n-1}\right)$. Thus we obtain

$$
V^{-1} H(n)^{k} V=D^{k} \text { or } V^{-1} G(n, k) V=D^{k},
$$

and so

$$
G(n, k)=V D^{k} V^{-1} .
$$

Consequently, after required computations, we get the following result:

Theorem 2. For $n, k>0$

$$
\begin{aligned}
(G(n, k))_{2,1} & =s(n, k) \\
& =\frac{(-1)^{n} U_{n(k-1)}+U_{n-1}^{k} U_{n}-U_{k n} U_{n-1}}{U_{n}^{3}} .
\end{aligned}
$$

From the definition of $s(n, k)$ and the above result, we have the following result:

Corollary 1. For $n, k>0$

$$
U_{n-1} U_{k n}=U_{n} U_{k n-1}+(-1)^{n} U_{n(k-1)} .
$$


Now we shall focus on the second quotient

$$
\frac{p^{k-1} U_{k n-2}-(-1)^{k+1} U_{n-2}^{k}}{U_{n}^{2}}
$$

and we will seek an explicit formula for that.

Similar to the process for the first quotient, we need some preliminary results and definition. First we define a second order recurrence $\left\{B_{n}(p)\right\}$, or briefly $B_{n}$, for $n>1$ as

$$
B_{n}=p B_{n-1}+B_{n-2}
$$

with initials $B_{0}=p^{2}-1$ and $B_{1}=p^{3}+2 p$.

For shortness, we denote the second quotient by $y(n, k)$ :

$$
y(n, k)=\frac{p^{k-1} U_{k n-2}-(-1)^{k+1} U_{n-2}^{k}}{U_{n}^{2}} .
$$

Define two matrices $T(n)$ and $Q(n, k)$ of order 3 as shown:

$$
T(n)=\left[\begin{array}{ccc}
B_{n-1} & C(n) & (-1)^{n+1} p^{2} U_{n-2} \\
1 & 0 & 0 \\
0 & 1 & 0
\end{array}\right]
$$

and

$$
Q(n, k)=\left[\begin{array}{lll}
y(n, k+2) & h(n, k+2) & (-1)^{n+1} p^{2} U_{n-2} y(n, k+1) \\
y(n, k+1) & h(n, k+1) & (-1)^{n+1} p^{2} U_{n-2} y(n, k) \\
y(n, k) & h(n, k) & (-1)^{n+1} p^{2} U_{n-2} y(n, k-1)
\end{array}\right],
$$

where $C(n)=p^{2}\left(D_{n}+2(-1)^{n}\right), D_{n}$ is defined as for $n>1$

$$
D_{n}=\left(p^{2}+2\right) D_{n-1}-D_{n-2}
$$

with initials $D_{0}=0$ and $D_{1}=1$, and, $h(n, k)$ is given by

$$
h(n, k)=\frac{p^{k-1} U_{n-2}^{2} U_{k n}-p^{k+1} U_{n(k-2)}-p(-1)^{k} U_{n-2}^{k} U_{2 n}}{U_{n}^{3}} .
$$

Lemma 2. For $n \geq 1$, the eigenvalues of $T(n)$ are $p \alpha^{n}, p \beta^{n}$ and $-U_{n-2}$.

Proof. The characteristic polynomial of $T(n)$ is $x^{3}-B_{n-1} x^{2}-C(n) x+(-1)^{n} p^{2} U_{n-2}$ and it is factorized as $\left(x-p \alpha^{n}\right)\left(x-p \beta^{n}\right)\left(x+U_{n-2}\right)$, as claimed.

We state our second main result:

Theorem 3. For $n \geq 1$,

$$
T(n)^{k}=Q(n, k) .
$$


Proof. To prove the claim, it is sufficient to verify the followings:

$$
y(n, k+1) B_{n-1}+h(n, k+1)=y(n, k+2)
$$

and

$$
y(n, k+1) C(n)+(-1)^{n+1} p^{2} U_{n-2} y(n, k)=h(n, k+2) .
$$

For proving them, one can consider the definitions of $y(n, k)$ and $h(n, k)$. The above two equations give us the following matrix equation:

$$
Q(n, k-1) T(n)=Q(n, k) .
$$

Since $Q(n, 1)=T(n)$, we deduce that $Q(n, k)=T(n)^{k}$.

Since all the eigenvalues of $T(n)$ are different from each other, it is diagonalized as

where

$$
V_{1}^{-1} T(n) V_{1}=D_{1},
$$

$$
V_{1}=\left[\begin{array}{ccc}
p^{2} \alpha^{2 n} & p^{2} \beta^{2 n} & U_{n-2}^{2} \\
p \alpha^{n} & p \beta^{n} & -U_{n-2} \\
1 & 1 & 1
\end{array}\right]
$$

and $D_{1}$ is the diagonal matrix $D_{1}=\operatorname{diag}\left(\alpha^{n}, \beta^{n},-U_{n-2}\right)$. Thus we obtain

$$
V_{1}^{-1} T(n)^{k} V_{1}=D_{1}^{k} \text { or } V_{1}^{-1} Q(n, k) V_{1}=D_{1}^{k},
$$

and so

$$
Q(n, k)=V_{1} D_{1}^{k} V_{1}^{-1} .
$$

Therefore, by considering $(Q(n, k))_{2,1}=y(n, k)$, we get the following result:

Theorem 4. For $n, k>0$

$$
y(n, k)=\frac{p^{k+1}(-1)^{n} U_{k n}+(-1)^{k+1} U_{n-2}^{k+1} U_{n}+p^{k} U_{n-2} U_{n(k+1)}}{U_{n}^{3}}
$$

From the definition of $y(n, k)$ and the above result, we have the following result:

Corollary 2. For $n, k>0$

$$
U_{n-2} U_{n k}=U_{n k-2} U_{n}-p(-1)^{n} U_{k n-n}
$$

\section{NEW RECURSIVE IDENTITIES}

While extending two curious properties in the previous section, we found the following two new identities for general second order recursions:

$$
\text { i) } \begin{aligned}
U_{n-1} U_{k n} & =U_{n} U_{k n-1}+(-1)^{n} U_{k n-n}, \\
\text { ii) } U_{n-2} U_{n k} & =U_{n} U_{k n-2}-p(-1)^{n} U_{k n-n} .
\end{aligned}
$$

The above recursions suggest us that they could be more generalized. Thus we give our third main result: 
Theorem 5. For all integers $r, k$ and $n$,

$$
U_{n-r}(p) U_{k n}(p)=U_{n}(p) U_{k n-r}(p)-(-1)^{r+n} U_{r}(p) U_{k n-n}(p)
$$

and

$$
U_{n-r}(p) V_{k n}(p)=U_{n}(p) V_{k n-r}(p)-(-1)^{r+n} U_{r}(p) V_{k n-n}(p)
$$

Proof. For the first identity consider

$$
\begin{aligned}
U_{n}(p) U_{k n-r}(p)-(-1)^{r+n} U_{r}(p) U_{k n-n}(p) \\
=\frac{\alpha^{n}-\beta^{n}}{\alpha-\beta} \frac{\alpha^{k n-r}-\beta^{k n-r}}{\alpha-\beta}-(-1)^{r+n} \frac{\alpha^{r}-\beta^{r}}{\alpha-\beta} \frac{\alpha^{k n-n}-\beta^{k n-n}}{\alpha-\beta} \\
=\left(\alpha^{k n+n-r}-(-1)^{n} \beta^{k n-n-r}-(-1)^{n} \alpha^{k n-n-r}+\beta^{k n+n-r}-(-1)^{n-r} \alpha^{k n-n+r}\right. \\
\left.\quad+(-1)^{n} \beta^{k n-n-r}+(-1)^{n} \alpha^{k n-n-r}-(-1)^{n-r} \beta^{k n-n+r}\right) /(\alpha-\beta)^{2} \\
=\left(\alpha^{k n+n-r}+\beta^{k n+n-r}-(-1)^{n-r} \alpha^{k n-n+r}-(-1)^{n-r} \beta^{k n-n+r}\right) /(\alpha-\beta)^{2} \\
\left.=\left(\alpha^{n-r}-\beta^{n-r}\right)\left(\alpha^{k n}-\beta^{k n}\right)\right) /(\alpha-\beta)^{2} \\
=U_{n-r}(p) U_{k n}(p),
\end{aligned}
$$

as claimed. By the Binet formula, the second claim could be similarly proved.

Our last conclusion is that the last recursive identities are new for general second order linear recurrences $\left\{U_{n}\right\}$ and $\left\{V_{n}\right\}$ according to our literature knowledge.

For the Fibonacci and Lucas cases, we have that

$$
\begin{aligned}
& \text { i) } F_{n-r} F_{k n}=F_{n} F_{k n-r}-(-1)^{n+r} F_{r} F_{k n-n} \text {, } \\
& \text { ii) } F_{n-r} L_{k n}=F_{n} L_{k n-r}-(-1)^{n+r} F_{r} L_{k n-n} \text {. }
\end{aligned}
$$

\section{Generalization OF THE DiVisibiLity PROPERTIES}

We consider two divisibility properties and then generalize them for the general second order linear recurrence $\left\{U_{n}\right\}$. Now we are ready to present more general case of these properties. We state this general case with the following corollary without proof:

Corollary 3. For all integers $r$,

$$
U_{r}^{k-1} U_{k n-r}-(-1)^{(r-1)(k+1)} U_{n-r}^{k} \text { is divisible by } U_{n}^{2}(n, k=1,2, \ldots) .
$$

This corollary gives us all the divisibility properties mentioned in this paper. 


\section{REFERENCES}

[1] M. Cavachi, "Some properties of the terms of the Fibonacci sequence," Gaz. Mat., Bucur., vol. 85, pp. 290-293, 1980.

[2] R. L. Graham, D. E. Knuth, and O. Patashnik, Concrete mathematics: A foundation for computer science. Addison-Wesley, 1992.

[3] E. Kiliç and P. Stănică, "A matrix approach for general higher order linear recurrences," Bull. Malays. Math. Sci. Soc. (2), vol. 34, no. 1, pp. 51-67, 2011.

[4] Y. V. Matiyasevich, "Enumerable sets are diophantine," Sov. Math., Dokl., vol. 11, pp. 354-358, 1970.

[5] S. Vajda, Fibonacci \& Lucas numbers, and the golden section. Theory and applications, ser. Ellis Horwood Books in Mathematics and its Applications. Chichester, New York: Ellis Horwood Ltd.; Halsted Press, 1989.

Author's address

\section{Emrah Kılıç}

TOBB Economics and Technology University, Mathematics Department 06560 Sogutozu Ankara Turkey.

E-mail address: ekilic@etu.edu.tr 